\title{
STRUCTURE AND STABILITY OF FLAMES WITH TWO SEQUENTIAL REACTIONS*
}

\author{
J. PELÁEZ† AND A. LIÑÁN†
}

\begin{abstract}
An asymptotic analysis is presented for the structure of steady planar flames, and for their response to nonplanar perturbations, when the reaction mechanism can be modelled by two sequential reactions with the same large value of the activation energies.

The analysis is carried out, with the thermal-diffusive approximation, for the distinguished "merged" regime, when the two reaction constants are such that the two reactions occur in a single thin zone. The nonlinear response of the flame can be described by a model involving only three parameters, associated with the Lewis number of the main reactant and the Lewis number and peak concentration, in the reaction zone, of the intermediate species.

A linear stability analysis of the planar flame suggests that close to the boundary of the domain of stability one can expect: chaotic response, cellular flames, travelling waves, pulsating flames or an instability associated with unsteady effects in the thin reaction zone.
\end{abstract}

1. Introduction. We present in this paper an analysis of the structure and stability of flames supported by a two-step sequential reaction mechanism of the form

$$
Y \stackrel{\text { (i) }}{\longrightarrow} \mu Z \stackrel{\text { (ii) }}{\longrightarrow} P
$$

under the assumption that both reactions are exothermic and have a high activation energy.

This scheme may result as a limiting form of some of the more general kinetic schemes, and represents also closely in some limiting cases, the simplified two step overall reaction mechanism proposed by Dryer and Glassman [1] and Westbrook and Dryer [2] for flames of hydrocarbon fuels in air,

$$
\begin{aligned}
& \mathrm{C}_{n} \mathrm{H}_{m}+\left(\frac{n}{2}+\frac{m}{4}\right) \mathrm{O}_{2} \rightarrow n \mathrm{CO}+\frac{m}{2} \mathrm{H}_{2} \mathrm{O}, \\
& \mathrm{CO}+\frac{1}{2} \mathrm{O}_{2} \rightleftarrows \mathrm{CO}_{2} .
\end{aligned}
$$

The previous model results when $\mathrm{O}_{2}$ is in excess, and the effect of the reverse reaction, that consumes $\mathrm{CO}_{2}$, is neglected; then $\mathrm{CO}$ acts as the intermediate species. The analytical methods used in this paper can be extended without major difficulties to analyse the cases where $\mathrm{O}_{2}$ is the limiting component, and to the cases where the effect of the reverse reaction is also taken into account.

When describing the structure and stability of flames that follow an overall kinetic scheme of the type (a) we shall use asymptotic techniques based on the fact that the activation energies of both reactions are large compared with the thermal energy.

Asymptotic techniques have been used previously to describe the structure of flames with simplified multi-step reaction mechanisms. The first important analysis were carried out by Zeldovich and co-workers to model chain reactions branching and nonbranching; see [3] and [4].

The flame structure for a two step sequential reaction mechanism was described for large activation energies by Berman and Riazantsev [5], who considered preexponential frequency factors of the same order, and found three different regimes, depending on the ratio $E_{1} / E_{2}$ of the activation energies and on the ratio $T_{1} / T_{b} . T_{1}$ is

* Received by the editors May 1, 1984, and in revised form October 1, 1984.

† Escuela Técnica Superior de Ingenieros Aeronáuticos, Universidad Politécnica de Madrid, Madrid, Spain. 
the flame temperature when the second reaction is neglected, and $T_{b}$ is the final flame temperature when the intermediate species has been completely consumed.

They found that if $E_{1}>E_{2}$ the second reaction is very fast compared with the first, so that the intermediate species is converted into products as soon as it is produced, and therefore it follows the steady state approximation. The flame velocity is determined by the rate of the first reaction, with a heat release that includes that of the second reaction.

For $1>T_{1} / T_{b}>E_{1} / E_{2}$ the second reaction is so slow that the flame velocity is determined by the heat release and rate of the first reaction. The temperature increases from its upstream value to $T_{1}$ across a transport zone that ends with a thin reaction zone, where $Y$ is consumed and $Z$ is produced. Downstream $Z$ is consumed in a long convective-reactive zone where the temperature grows to its final value $T_{b}$.

If $1>E_{1} / E_{2}>T_{1} / T_{b}$, the flame velocity associated with the second reaction at the temperature $T_{b}$ is higher than that of the first reaction (with a final temperature $T_{1}$ ) so that the trailing convective-reactive zone of the previous case does not exist here. In this case, the flame structure shows the two reactions occurring in separate thin reaction zones; the first reaction zone is embedded in the transport zone of the second reaction.

This analysis was extended by Kapila and Ludford [6] to the more general cases where the frequency factors are not of the same order. They recovered the three regimes mentioned above, with the transition value of $E_{1} / E_{2}$ depending now on the ratio of the frequency factors.

There is a distinguished transition regime, that we shall call "merged" regime, between the regime of quasi-steady state approximation of the intermediate and the regime of separate thin reaction zones, for which the two reactions occur in a single thin reaction zone. Kapila and Ludford [6] considered briefly the merged regime in the limiting cases $E_{1} / E_{2} \gg 1$ or $E_{1} / E_{2} \ll 1$.

Joulin and Clavin [7] had carried out previously an analysis of the structure of the flames in this regime for the case where the two activation energies are equal and the first reaction has a negligible heat release.

Margolis and Matkowsky [8] analysed the merged regime for the case of roughly equal activation energies and a particular value of the ratio of the heat release of the two reactions that lead to a simplified flame structure.

All the previous analyses have been limited to the study of the quasi-steady planar flame structure. An analysis of stability of planar flames, under planar perturbation, was carried out by Margolis and Matkowsky [9] for the particular case of equal activation energies and a special value of the ratio of heat release. However, they considered that the Lewis number of the intermediate species was close to one, and as a consequence they recovered the stability properties of the one-step Arrhenius reaction.

In this paper we extend their analysis of the steady planar flame structure and its stability under nonplanar perturbations, in the merged regime, for arbitrary values of the heat release of the two reactions for the interesting case in which the Lewis number of the intermediate species is not close to one. We shall be concerned mainly with the case where the two activation energies are large, with a ratio $E_{2} / E_{1}$ equal to 1 . The case $E_{2} / E_{1} \neq 1$ and the analysis of the stability of the flame in the regime of separate flames will be considered in a separate paper.

Section 2 is devoted to the formulation of the problem, within the framework of the thermal-diffusive approximation: that is when the flow velocities induced by changes in density are neglected. 
The steady planar flame structure is described in $\S 3$, for values of the rate constants leading to the merged regime. The concentration of the intermediate is small in this regime; it decreases to zero at the downstream edge of the thin reaction zone, and takes its peak value just upstream of the zone. This peak value is obtained together with the reactant burning rate per unit flame surface, as a function of the flame temperature, from the analysis of the reaction zone.

In $\S 4$ we derive, using the results of the previous section, a model that describes for large activation energies the nonlinear transient response of the flame in the merged regime when subject to nonplanar perturbations. It is a generalization to the sequential mechanism of a model derived by Matkowsky and Sivashinsky [10] for one-step Arrhenius kinetics. Only three parameters are involved in this model; namely, the Lewis number of the intermediate species, the deviation of the Lewis number of the main reactant from unit times the nondimensional activation energy, and a nondimensional measure of the peak concentration of the intermediate. We use the model in $\S 5$ to analyse the linear stability of the steady planar flame front structure, and thus display the effects of the multiple-kinetics on the response of the flame close to the stability limit. A short summary of the result is given in $\S 5$.

2. Formulation. We begin by writing the conservation equations for the diffusional-thermal model, for which the fluid dynamic effects associated with density changes due to thermal expansion are neglected, so that no motion is induced by the flame propagation process. We shall also consider that, together with the density, the mixture specific heat $c_{p}$, the thermal conductivity $\lambda$, and the diffusion coefficients $D_{Y}, D_{Z}$ of the species $Y$ and $Z$ in the mixture, are constant.

For a reference system that sees the unreacted medium at rest, the mass and energy conservation equations for the mass fractions $\bar{Y}, \bar{Z}$ and the temperature $\bar{T}$ take the form

$$
\begin{aligned}
& \frac{\partial \bar{Y}}{\partial \bar{t}}=D_{Y}\left(\frac{\partial^{2} \bar{Y}}{\partial \bar{x}^{2}}+\frac{\partial^{2} \bar{Y}}{\partial \bar{y}^{2}}\right)-W_{Y} \hat{\omega}_{1} / \rho, \\
& \frac{\partial \bar{Z}}{\partial \bar{t}}=D_{Z}\left(\frac{\partial^{2} \bar{Z}}{\partial \bar{x}^{2}}+\frac{\partial^{2} \bar{Z}}{\partial \bar{y}^{2}}\right)+\frac{\mu W_{Z} \hat{\omega}_{1}}{\rho}-\frac{W_{Z} \hat{\omega}_{2}}{\rho}, \\
& \frac{\partial \bar{T}}{\partial \bar{t}}=\frac{\lambda}{\rho c_{p}}\left(\frac{\partial^{2} \bar{T}}{\partial \bar{x}^{2}}+\frac{\partial^{2} \bar{T}}{\partial \bar{y}^{2}}\right)+\frac{q_{1}}{c_{p}} \frac{\hat{\omega}_{1}}{\rho}+\frac{q_{2}}{c_{p}} \frac{\hat{\omega}_{2}}{\rho},
\end{aligned}
$$

where we have not included, for simplicity in the presentation, derivatives with respect to the third spacial coordinate.

Here $\hat{\omega}_{1}$ and $\hat{\omega}_{2}$ are the molar production rates due to the reactions (i) and (ii), and we assume that they follow an Arrhenius law of the type

$$
\begin{aligned}
& \hat{\omega}_{1}=B_{1}[\mathrm{O}]^{\nu_{1}}[Y] \exp \left(-E_{1} / R \bar{T}\right), \\
& \hat{\omega}_{2}=B_{2}[\mathrm{O}]^{\nu_{2}}[Z] \exp \left(-E_{2} / R \overline{\bar{T}}\right),
\end{aligned}
$$

involving frequency factors $B_{i}$ and activation energies $E_{i} . W_{Y}$ and $W_{Z}$ are the molecular mass of $Y$ and $Z, \mu$ is a stoichiometric coefficient and $q_{1}$ and $q_{2}$ are the molar heat of the reactions (i) and (ii). We shall also consider that the oxidizer molar concentration [O] is large compared with that of the species $Y$ and $Z$, so that we shall neglect the variation of [O] in (4) and (5).

The analysis will be carried out for a reaction order equal to 1 , with respect to $Y$ and $Z$, although it can be generalized without major difficulties to other reaction orders. 
The system of equations (1)-(3) has wave front solutions corresponding to planar steady propagating flame fronts with velocity $U_{F}$, in the $-\bar{x}$ direction for example, such that the concentrations and temperature are functions of the variable $\bar{x}+U_{F} t$.

For large values of the activation energies $E_{1}$ and $E_{2}$, the reactions take place in thin reaction layers, one of them in the end of a thicker transport layer and the other embedded in this transport layer. We shall be concerned in this paper with a distinguished regime corresponding to a certain range of values of the ratio of the frequency factors $B_{1} / B_{2}$, that we shall specify below, for which the two thin reaction layers merge into a thin reaction zone that follows the transport zone. When the flame is seen with the characteristic length of the transport zone, the reaction layer appears as an infinitely thin flame sheet, in the limit of large values of the nondimensional activation energies. The chemical reaction is frozen in the upstream transport zone.

The solution of the system of equations (1)-(3) must satisfy the boundary conditions

$$
\begin{aligned}
& \bar{x} \rightarrow-\infty: \quad \bar{Y}=\bar{Y}_{u}, \quad \bar{Z}=0, \quad \bar{T}=\bar{T}_{u}, \\
& \bar{x} \rightarrow+\infty, \quad \bar{Y}=0, \quad \bar{Z}=0, \quad \bar{T}=\bar{T}_{b},
\end{aligned}
$$

for a certain flame velocity $U_{F}$ that appears as an eigenvalue.

Here the subscript $u$ refers to the unburned upstream conditions that are considered uniform and $\bar{T}_{b}$ is the final, adiabatic, flame temperature, given by

$$
\bar{T}_{b}=\bar{T}_{u}+\left(q_{1}+\mu q_{2}\right) \bar{Y}_{u} / c_{p} W_{Y}
$$

The conditions $\bar{Y}=0, \bar{Z}=0$ on the hot side of the flame correspond to the chemical equilibrium state associated with the simplified scheme (i)-(ii).

We shall be concerned with the analysis of the structure of the planar steady flame fronts, and of their stability to transverse perturbations, taking advantage of the fact that the activation energies are large compared with the thermal energy $R \bar{T}_{b}$. In this case the reaction zone appears as a flame sheet, in the limit $E / R \bar{T}_{b} \rightarrow \infty$, located at

$$
\bar{x}_{F}=-U_{F} \bar{t}+\bar{\phi}(\bar{y}, \bar{t}),
$$

where $\bar{\phi}(\bar{y}, \bar{t})$ represents the flame displacement, from its steady planar location, due to perturbations.

For the description of the flame structure we shall use the variables $\bar{t}, \bar{y}$ and

$$
\xi=\bar{x}-\bar{x}_{F}=\bar{x}+U_{F} \bar{t}-\bar{\phi}(\bar{y}, \bar{t})
$$

or their nondimensional form $t, y$ and $x$, based on the thickness of the transport zone $l_{T}=\lambda / \rho U_{F} c_{p}$, as the unit of length, and $l_{T} / U_{F}$ as the unit of time. As dependent variables we shall use $Y=\bar{Y} / \bar{Y}_{u}, Z=\bar{Z} W_{Y} /\left(\mu W_{z} \bar{Y}_{u}\right), \theta=\left(\bar{T}-\bar{T}_{u}\right) /\left(\bar{T}_{b}-\bar{T}_{u}\right)$ and the nondimensional flame displacement $\phi(y, t)=\bar{\phi} / l_{T}$.

The conservation equations take the form

$$
\begin{aligned}
& \frac{\partial Y}{\partial t}+\left(1-\frac{\partial \phi}{\partial t}\right) \frac{\partial Y}{\partial x}=\frac{1}{L_{Y}} \Delta Y-\beta^{2} \Lambda_{1} \frac{Y}{L_{Y}} \exp \left\{\frac{\beta(\theta-1)}{1+\gamma(\theta-1)}\right\}, \\
& \frac{\partial Z}{\partial t}+\left(1-\frac{\partial \phi}{\partial t}\right) \frac{\partial Z}{\partial x}=\frac{1}{L_{Z}} \Delta Z+\beta^{2} \Lambda_{1}\left(\frac{Y}{L_{Y}}-K \frac{Z}{L_{Z}}\right) \exp \left\{\frac{\beta(\theta-1)}{1+\gamma(\theta-1)}\right\}, \\
& \frac{\partial \theta}{\partial t}+\left(1-\frac{\partial \phi}{\partial t}\right) \frac{\partial \theta}{\partial x}=\Delta \theta+\beta^{2} \Lambda_{1}\left(\frac{\alpha Y}{L_{Y}}+(1-\alpha) K \frac{Z}{L_{Z}}\right) \exp \left\{\frac{\beta(\theta-1)}{1+\gamma(\theta-1)}\right\},
\end{aligned}
$$


where $\Delta$ represents the operator

$$
\frac{\partial^{2}}{\partial x^{2}}+\left(\frac{\partial}{\partial y}-\frac{\partial \phi}{\partial y} \frac{\partial}{\partial x}\right)^{2} .
$$

The boundary conditions are

$$
\begin{aligned}
& x \rightarrow-\infty: \quad Y=1, \quad Z=0, \quad \theta=0, \\
& x \rightarrow+\infty: \quad Y=0, \quad Z=0, \quad \theta=1 \text {. }
\end{aligned}
$$

Here $L_{Y}=\lambda / \rho D_{Y} c_{p}$ and $L_{Z}=\lambda / \rho D_{Z} c_{p}$ are the Lewis numbers of the reacting species $Y$ and $Z, \alpha=q_{1} /\left(q_{1}+\mu q_{2}\right)$ is the fraction of the total heat release coming from the first reaction. We shall consider both reactions to be exothermic and therefore $0<\alpha<1$.

For simplicity in the presentation we have also considered both reactions to have the same activation energy $E_{2}=E_{1}$, although the analysis can be generalized to different values of the activation energies. The nondimensional parameter $\gamma=\left(\bar{T}_{b}-\bar{T}_{u}\right) / \bar{T}_{b}$ is, in practical cases, a number of order unity; for the validity of the thermal diffusive model this number should be very small compared with unity, as shown by Matkowsky and Sivashinsky [10].

The Zeldovich number

$$
\beta=\frac{E_{1}}{R T_{b}} \gamma=\frac{E_{1}}{R T_{b}} \frac{\bar{T}_{b}-\bar{T}_{u}}{\bar{T}_{b}} \gg 1
$$

will be considered to be large, so that the reaction is confined to a thin layer.

$\Lambda_{1}$ is an eigenvalue, $\sim U_{F}^{-2}$, defined by

$$
\Lambda_{1}=\frac{L_{Y}}{\beta^{2}} \frac{B_{1}[\mathrm{O}]^{\nu_{1}} \rho \lambda}{c_{p}\left(\rho U_{F}\right)^{2}} \exp \left(-\frac{E_{1}}{R \bar{T}_{b}}\right),
$$

to be determined as part of the solution of the steady planar front propagation problem in terms of the parameter

$$
K=\frac{L_{Z}}{L_{Y}} \frac{B_{2}}{B_{1}}[\mathrm{O}]^{\nu_{2}-\nu_{1}}
$$

that measures the ratio of the reactions rates of the reactions.

In the merged regime, that we shall consider, this number $K$ is of order unity. The analysis for the merged regime presented below applies also to the cases where $K \gg 1$. However in the opposite limiting case, where $K$ becomes very small compared to 1 , the two reactions occur in separate thin reactions layers, and the results given below become only qualitative if the separation of the reaction zones is too large.

3. Steady planar flame propagation. The conservation equations reduce in this case to the system

$$
\begin{aligned}
& \frac{d Y}{d x}-\frac{1}{L_{Y}} \frac{d^{2} Y}{d x^{2}}=-\Lambda_{1} \beta^{2} \frac{Y}{L_{Y}} \exp \left(\frac{\beta(\theta-1)}{1+\gamma(\theta-1)}\right), \\
& \frac{d Z}{d x}-\frac{1}{L_{Z}} \frac{d^{2} Z}{d x^{2}}=\beta^{2} \Lambda_{1}\left(\frac{Y}{L_{Y}}-K \frac{Z}{L_{Z}}\right) \exp \left(\frac{\beta(\theta-1)}{1+\gamma(\theta-1)}\right), \\
& \frac{d \theta}{d x}-\frac{d^{2} \theta}{d x^{2}}=\beta^{2} \Lambda_{1}\left(\alpha \frac{Y}{L_{Y}}+(1-\alpha) K \frac{Z}{L_{Z}}\right) \exp \left(\frac{\beta(\theta-1)}{1+\gamma(\theta-1)}\right)
\end{aligned}
$$

with the boundary conditions (14). 
In the limit $\beta \rightarrow \infty$ the reaction terms in the right-hand side of (18)-(20) are exponentially small outside an infinitely thin layer, that we shall place at $x=0$, where the temperature $\theta$ differs from 1 by an amount $1 / \beta$.

So in the outer convective-diffusive regions the concentration and temperature are given by

$$
\begin{array}{ll}
x<0: & Y=1-\exp \left(x L_{Y}\right), \quad Z=A \exp \left(x L_{Z}\right) \quad \theta=\exp (x-H), \\
x>0: \quad Y=0, \quad Z=0, \quad \theta=1 .
\end{array}
$$

The origin of the coordinate system has been placed at the position where the main reactant concentration $Y$, as given by the upstream outer solution, is zero, see Fig. 1. We anticipate the existence of jumps $-A$ and $1-\exp (-H)$, at the flame sheet,

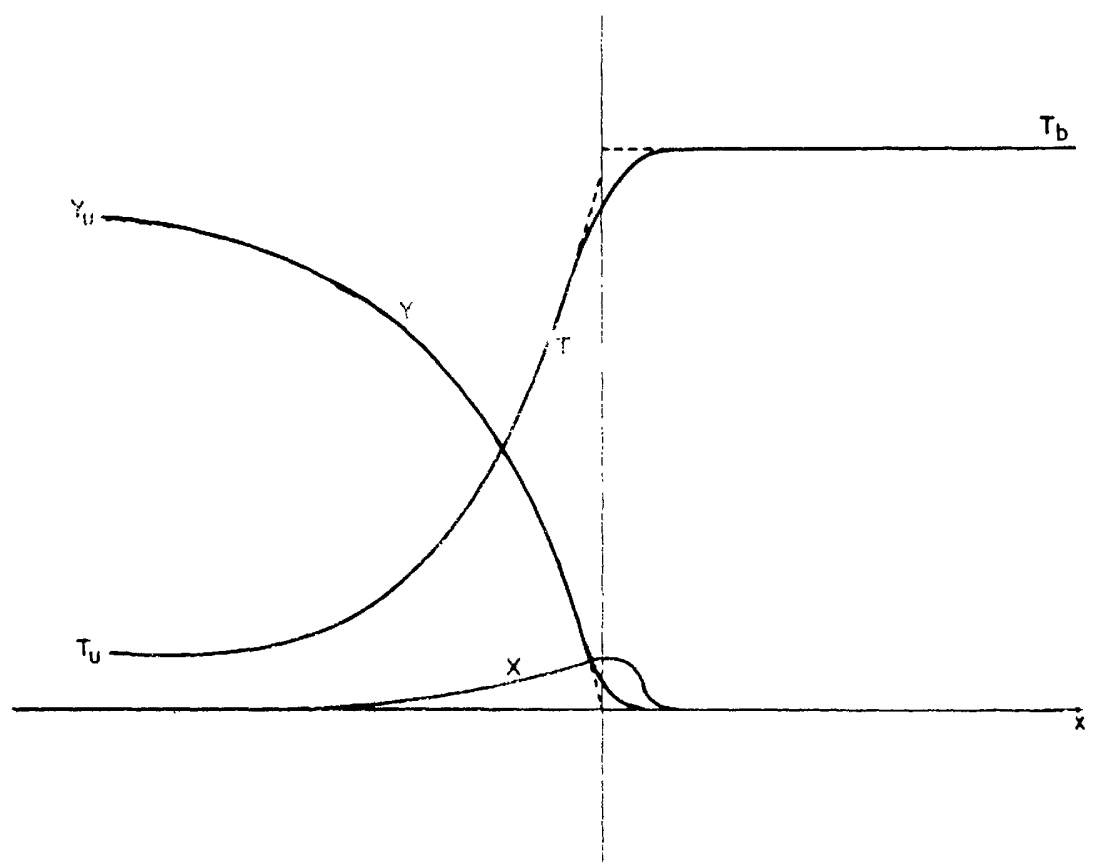

FIG. 1. Temperature and concentration profiles of the steady planar flame.

in the values of $Z$ and $\theta$ given by the outer solutions; these will be found in the form of expansions in powers of $1 / \beta$ of the form

$$
A=\frac{n L_{Z}}{\beta}+\cdots \quad \text { and } \quad H=\frac{h}{\beta}+\cdots
$$

where $h$ and $n$ are, of order unity, to be determined below.

Reaction zone structure. In order to describe the structure of the reaction layer we shall use an inner variable

$$
\eta=\beta x
$$

of order unity in the reaction zone.

We shall write the solution in the form of expansions

$$
\begin{aligned}
& \Lambda_{1}=\Lambda+\beta^{-1} \Lambda^{(1)}+\cdots, \\
& Y=L_{Y}\left(\tilde{Y}_{1} \beta^{-1}+\tilde{Y}_{2} \beta^{-2}+\cdots\right),
\end{aligned}
$$




$$
\begin{aligned}
& Z=L_{Z}\left(\tilde{Z}_{1} \beta^{-1}+\tilde{Z}_{2} \beta^{-2} \cdots\right), \\
& \theta=1+\tilde{\theta}_{1} \beta^{-1}+\tilde{\theta}_{2} \beta^{-2}+\cdots,
\end{aligned}
$$

where $\tilde{Y}_{1}, \tilde{Y}_{2}, \cdots$ are functions of $\eta$. From (18)-(20) we obtain the system

$$
\begin{aligned}
& \frac{d^{2} \tilde{Y}_{1}}{d \eta^{2}}=\Lambda \tilde{Y}_{1} \exp \left(\tilde{\theta}_{1}\right), \\
& \frac{d^{2} \tilde{Z}_{1}}{d \eta^{2}}=\Lambda\left(K \tilde{Z}_{1}-\tilde{Y}_{1}\right) \exp \left(\tilde{\theta}_{1}\right), \\
& \frac{d^{2} \tilde{\theta}_{1}}{d \eta^{2}}=-\Lambda\left(\alpha \tilde{Y}_{1}+(1-\alpha) K \tilde{Z}_{1}\right) \exp \left(\tilde{\theta}_{1}\right)
\end{aligned}
$$

that respresents a diffusive-reactive balance, with the two reactions playing a similar role in the reaction zone.

The boundary conditions

$$
\begin{array}{llll}
\eta \rightarrow-\infty: & \tilde{Y}_{1}+\eta \rightarrow 0, & \tilde{Z}_{1} \rightarrow n, & \tilde{\theta}_{1}-\eta+h \rightarrow 0, \\
\eta \rightarrow+\infty: & \tilde{Y}_{1} \rightarrow 0, & \tilde{Z}_{1} \rightarrow 0, & \tilde{\theta}_{1} \rightarrow 0
\end{array}
$$

are obtained from the matching conditions with the outer solution (21).

From the conservation equations (28)-(30), when the boundary conditions ( $31 \mathrm{~b})$ are taken into account, we obtain the relation

$$
\tilde{Y}_{1}+\tilde{\theta}_{1}+(1-\alpha) \tilde{Z}_{1}=0
$$

that will be used below together with two of the equations (28)-(30).

From (32) and the boundary conditions (31a) we obtain the relation

$$
h=(1-\alpha) n,
$$

between the apparent jumps in $\theta$ and $Z$ at the flame sheet.

The system of (28), (30), (32), with the boundary condition (31), can be solved numerically to obtain in particular the eigenvalues $\Lambda$ and $h$ as functions of $K$ and $\alpha$. The details of this analysis are given in the Appendix, and the results are summarized in Figs. 2 and 3. The particular case $\alpha=0$ was integrated numerically by Joulin and Clavin [7] who computed $\Lambda(K)$. In the special case $K=\alpha$ considered by Margolis and Matkowsky [8], the problem of determining $\Lambda(K)$ is simplified because when (32) is taken into account, (30) reduces to

$$
\frac{d^{2} \tilde{\theta}_{1}}{d \eta^{2}}=\Lambda K \tilde{\theta}_{1} e^{\tilde{\theta}_{1}}
$$

that, when solved with the boundary conditions (31), yields

$$
\Lambda=\frac{1}{2 K} \text { for } \alpha=K
$$

while (29) can be solved numerically to calculate $h(K)$. In [8], a series expansion for $h$ was presented.

4. Limiting model for nonplanar unsteady flame propagation. We have carried out a linear stability analysis of the steady planar flame to nonplanar perturbation, in the merged regime, for large $\beta$, similar to that carried out by Joulin and Clavin [13] for the one-step Arrhenius kinetics, to show that the planar flame is unstable if the Lewis 


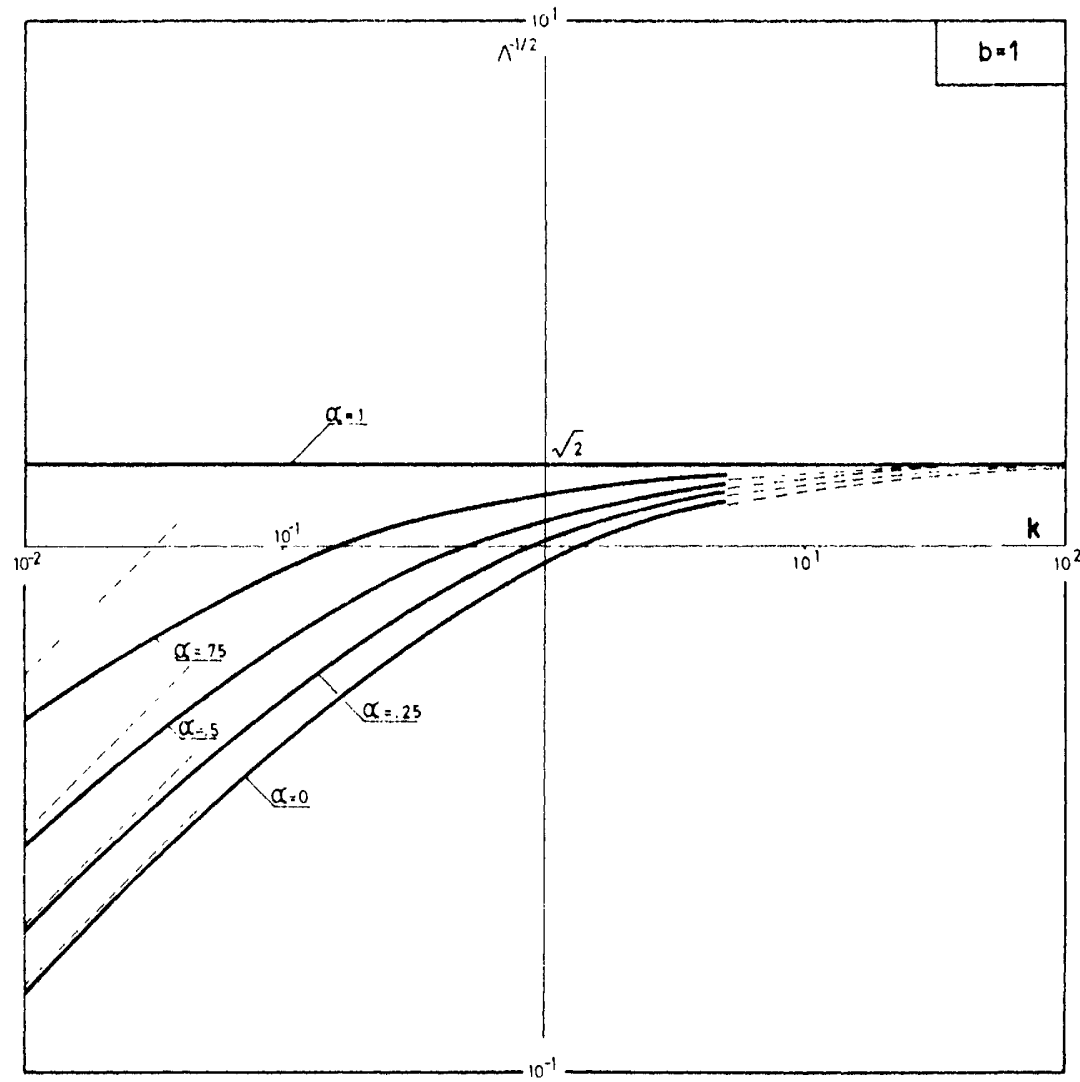

FIG. 2. The flame velocity eigenvalue $\Lambda$ versus $K$ for several values of $\alpha$. Solid lines correspond to numerical results; dotted lines...- correspond to the asymptotic behaviour for small or large value of $K$.

number $L_{Y}$ of the main reactant is different from 1 . In fact the following dispersion relation

$$
1-L_{Y}=\sqrt{1+4\left(\omega+k^{2}\right)}-\sqrt{L_{Y}^{2}+4\left(\omega L_{Y}+k^{2}\right)}
$$

is obtained between the amplification rate $\omega$ of the disturbance, its transverse wave number $k$ and $L_{Y}$. (36) has the root $\omega=k$, and therefore the flame is unstable for all values of $L_{Y} \neq 1$. For $L_{Y}=1$ the relation (36) is satisfied identically for all values of $\omega$ and $k$.

Thus the results of this analysis indicates that the flame can only be stable if the Lewis number does not differ significantly from 1 . As we shall see below, the stable range is confined to values of $L_{Y}-1=l_{e} / \beta$, with $l_{e}$ of order unity.

In the following we shall derive from (11)-(13), in the limit of large $\beta$ with $l_{e}$ of order unity, a simplified model for the unsteady nonplanar flame propagation, that represents a generalization for the sequential reaction mechanism, in the merged regime, of the model obtained by Matkowsky and Sivashinsky [10] for one-step Arrhenius kinetics.

Notice first that from the conservation equations (11)-(13) we can derive the equation

$$
\left(\frac{\partial}{\partial t}+\left(1-\frac{\partial \phi}{\partial t}\right) \frac{\partial}{\partial x}\right)(Y+\theta+(1-\alpha) Z)=\Delta\left(\frac{Y}{L_{Y}}+\theta+(1-\alpha) \frac{Z}{L_{Z}}\right),
$$

free from the reaction terms, that we shall use in the following to replace (13). 


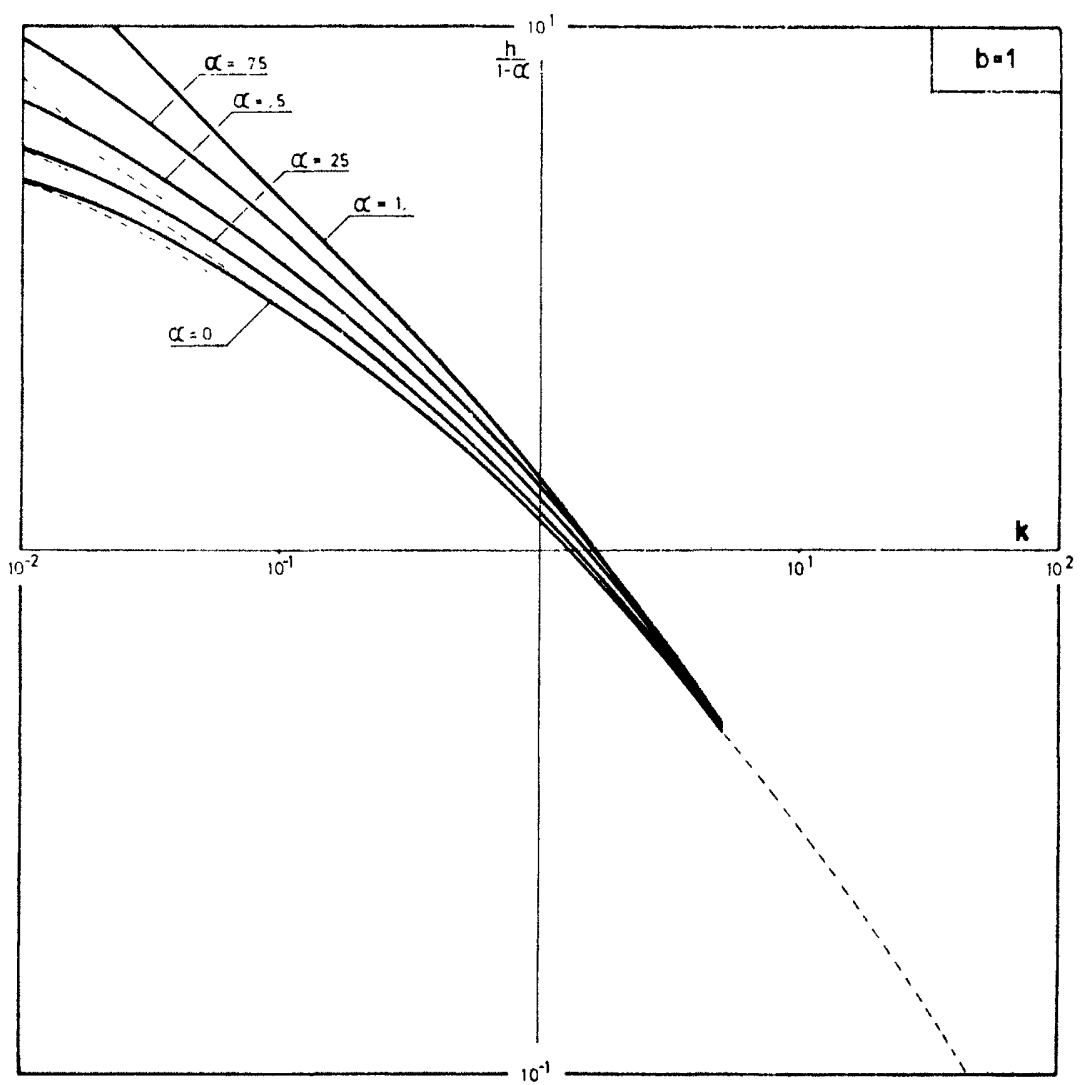

FIG. 3. The nondimensional peak value of the intermediate concentration as a function of $K$. Solid lines - correspond to numerical results; dotted lines .... - correspond to the asymptotic expressions for small and large values of $K$.

As indicated before, for values of $\beta \gg 1$, the reaction, in the merged regime, is confined to a thin zone, of thickness $1 / \beta$ relative to that of the outer preheat zone. In the outer regions, upstream and downstream of the reaction zone, we shall describe the solution in terms of the expansions

$$
\begin{aligned}
& Y=Y_{0}+\beta^{-1} Y_{1}+\cdots, \\
& Z=\beta^{-1} Z_{1}+\cdots, \\
& \theta=\theta_{0}+\beta^{-1} \theta_{1}+\cdots, \\
& \phi=\phi_{0}+\beta^{-1} \phi_{1}+\cdots .
\end{aligned}
$$

When these expansions are introduced in (11)-(12)-(37), we obtain the following system of equations and boundary conditions:

$$
\begin{aligned}
& \frac{\partial Y_{0}}{\partial t}+\left(1-\frac{\partial \phi_{0}}{\partial t}\right) \frac{\partial Y_{0}}{\partial x}=\Delta Y_{0}, \\
& \left(\frac{\partial}{\partial t}+\left(1-\frac{\partial \phi_{0}}{\partial t}\right) \frac{\partial}{\partial x}\right)\left(Y_{0}+\theta_{0}\right)=\Delta\left(Y_{0}+\theta_{0}\right), \\
& x \rightarrow-\infty: \quad Y_{0}=1, \quad Y_{0}+\theta_{0}=1, \\
& x \rightarrow+\infty: \quad Y_{0}=0, \quad Y_{0}+\theta_{0}=1,
\end{aligned}
$$




$$
\begin{aligned}
& \left(\frac{\partial}{\partial t}+\left(1-\frac{\partial \phi_{0}}{\partial t}\right) \frac{\partial}{\partial x}\right)\left(Y_{1}\right)=\Delta Y_{1}-l_{e} \Delta Y_{0}+\frac{\partial \phi_{1}}{\partial t} \frac{\partial Y_{0}}{\partial x}+\tilde{\Delta} Y_{0}, \\
& \left(\frac{\partial}{\partial t}+\left(1-\frac{\partial \phi_{0}}{\partial t}\right) \frac{\partial}{\partial x}\right)\left(Z_{1}\right)=\frac{1}{L_{Z}} \Delta Z_{1}, \\
& \left(\frac{\partial}{\partial t}+\left(1-\frac{\partial \phi_{0}}{\partial t}\right) \frac{\partial}{\partial x}\right)\left\{Y_{1}+\theta_{1}+(1-\alpha) Z_{1}\right\} \\
& =-l_{e} \Delta Y_{0}+\Delta\left(Y_{1}+\theta_{1}+(1-\alpha) \frac{Z_{1}}{L_{Z}}\right)+\frac{\partial \phi_{1}}{\partial t} \frac{\partial}{\partial x}\left(Y_{0}+\theta_{0}\right)+\tilde{\Delta}\left(Y_{0}+\theta_{0}\right), \\
& x \rightarrow-\infty: \quad Y_{1}=Z_{1}=\theta_{1}=0, \\
& x \rightarrow+\infty: \quad Y_{1}=Z_{1}=\theta_{1}=0
\end{aligned}
$$

where $\Delta$ and $\tilde{\Delta}$ are differential operators given by

$$
\Delta \equiv \frac{\partial}{\partial x^{2}}+\left(\frac{\partial}{\partial y}-\frac{\partial \phi_{0}}{\partial y} \frac{\partial}{\partial x}\right)^{2}, \quad \tilde{\Delta} \equiv 2 \frac{\partial \phi_{0}}{\partial y} \frac{\partial \phi_{1}}{\partial y} \frac{\partial^{2}}{\partial x^{2}}-2 \frac{\partial \phi_{1}}{\partial y} \frac{\partial^{2}}{\partial x \partial y}-\frac{\partial^{2} \phi_{1}}{\partial y^{2}} \frac{\partial}{\partial x} .
$$

The reaction zone is placed at $x=0$, and as seen with the outer scale appears as infinitely thin. Downstream of the reaction zone the reactants are depleted so that $Y_{0}=Y_{1}=Z_{1}=0$ for $x>0$.

We should add appropriate initial conditions to these equations. Our main objective is the study of the evolution of perturbations of a steady planar flame. We shall hence consider initial conditions representing a small perturbation of the planar structure. If we notice that for the steady planar flame $Y_{0}+\theta_{0}=1$, we shall consider initial conditions such that

$$
(Y+\theta)_{t=0}=1+O\left(\beta^{-1}\right)
$$

and hence $Y_{0}+\theta_{0}=1$ at $t=0$. Therefore the solution of $(40)$ is

$$
Y_{0}+\theta_{0}=1 \text {, }
$$

and the last term in (45) drops out of the equation.

For the solution of (39)-(47) we need also jump conditions at $x=0$, that must be obtained from the matching conditions with the solution for the inner reaction zone. For the analysis of this zone we use as variables $y$ and $t$, with a stretched inner variable

$$
\eta=\beta x
$$

and the following expansions

$$
\begin{aligned}
& Y=\beta^{-1} \hat{Y}_{1}+\beta^{-2} \hat{Y}_{2}+\cdots, \\
& Z=\beta^{-1} \hat{Z}_{1}+\beta^{-2} \hat{Z}_{2}+\cdots, \\
& \theta=1+\beta^{-1} \hat{\theta}_{1}+\beta^{-2} \hat{\theta}_{2}+\cdots, \\
& \phi=\phi_{0}+\beta^{-1} \phi_{1}+\cdots
\end{aligned}
$$

where $\hat{Y}_{1}, \hat{Y}_{2}$, etc. are functions of $\eta, y$ and $t$.

The eigenvalue $\Lambda_{1}$ is fixed in this analysis of the transient response of the flame. Its value was calculated by means of the expansion (24) in the analysis of the steady flame propagation process, that resulted in $\Lambda_{1}=\Lambda(K, \alpha)$ in first approximation. 
When the expansions (50) are used together with the conservation equations (11)-(12)-(37) we obtain the following system of equations

$$
\begin{aligned}
& \left(1+\left(\frac{\partial \phi_{0}}{\partial y}\right)^{2}\right) \frac{\partial^{2} \hat{Y}_{1}}{\partial \eta^{2}}=\Lambda \hat{Y}_{1} e^{\hat{\theta}_{1}} \\
& \left(1+\left(\frac{\partial \phi_{0}}{\partial y}\right)^{2}\right) \frac{\partial^{2} \hat{\theta}_{1}}{\partial \eta^{2}}=-\Lambda\left(\alpha \hat{Y}_{1}+(1-\alpha) K \frac{\hat{Z}_{1}}{L_{Z}}\right) e^{\hat{\theta}_{1}} \\
& \frac{\partial^{2}}{\partial \eta^{2}}\left(\hat{Y}_{1}+\hat{\theta}_{1}+(1-\alpha) \frac{\hat{Z}_{1}}{L_{Z}}\right)=0
\end{aligned}
$$

to be solved with the boundary conditions

$$
\begin{aligned}
& \eta \rightarrow-\infty:\left\{\begin{array}{l}
\hat{Y}_{1} \rightarrow \frac{\partial Y_{0}}{\partial x}\left(0^{-}, y, t\right) \eta, \quad \hat{Z}_{1} \rightarrow Z_{1}\left(0^{-}, y, t\right), \\
\hat{\theta}_{1} \rightarrow \frac{\partial \theta_{0}}{\partial x}\left(0^{-}, y, t\right) \eta+\theta_{1}\left(0^{-}, y, t\right),
\end{array}\right. \\
& \eta \rightarrow+\infty: \quad \hat{Y}_{1} \rightarrow 0, \quad \hat{Z}_{1} \rightarrow 0, \quad \hat{\theta}_{1} \rightarrow \theta_{1}\left(0^{+}, y, t\right)
\end{aligned}
$$

obtained from the matching conditions with the outer expansions. The first of the boundary conditions (54) is a consequence of the choice of the thin reaction zone location, as the surface where the concentration $Y$ of the main reactant, as given by the outer solution, becomes zero.

Notice that, in first approximation, the structure of the reaction zone is quasisteady, as corresponds to the assumption that the transients are associated with the characteristic response time of the outer transport zone. In fact this structure, as it will be shown below, is identical to that of the steady planar flame.

From (53)-(55) we obtain the conservation equation

$$
\hat{Y}_{1}+\hat{\theta}_{1}+(1-\alpha) \frac{\hat{Z}_{1}}{L_{Z}}=\theta_{1}\left(0^{+}, y, t\right)
$$

The term $\theta_{1}\left(0^{+}, y, t\right)$ in the right-hand side of $(56)$ reflects the fact that due to unsteady and nonplanar perturbations the temperature just downstream of the reaction zone differs from the adiabatic flame temperature.

As a consequence of (56) the following relation,

$$
\left[\theta_{1}\right]+\frac{1-\alpha}{L_{Z}}\left[Z_{1}\right]=0
$$

is obtained between the jumps, $\left[\theta_{1}\right]=\theta_{1}\left(0^{+}, y, t\right)-\theta_{1}\left(0^{-}, y, t\right)$ and $\left[Z_{1}\right]=-Z_{1}\left(0^{-}, y, t\right)$, of the outer perturbations $\theta_{1}$ and $Z_{1}$, at the thin reaction zone, if we take into account that, from the definition of the thin flame position, there are not jumps of $Y$ at the thin reaction zone

$$
\left[Y_{0}\right]=\left[Y_{1}\right]=0
$$

By using the change of variables

$$
\zeta=-\frac{\partial Y_{0}}{\partial x}\left(0^{-}, y, t\right) \eta, \quad u=\hat{\theta}_{1}-\theta_{1}\left(0^{+}, y, t\right)
$$


and (56), we can write (51) and (52) in the form

$$
\begin{gathered}
\frac{\partial^{2} \hat{Y}_{1}}{\partial \zeta^{2}}=\lambda_{0} \hat{Y}_{1} e^{u}, \\
\frac{\partial^{2} u}{\partial \zeta^{2}}=\lambda_{0}\left\{\hat{Y}_{1}(K-\alpha)+K u\right\} e^{u}
\end{gathered}
$$

where $\lambda_{0}$ is given by

$$
\lambda_{0}=\Lambda e^{\theta_{1}\left(0^{+}, y, t\right)}\left\{\frac{\partial Y_{0}}{\partial x}\left(0^{-}, y, t\right)\right\}^{-2}\left\{1+\left(\frac{\partial \phi_{0}}{\partial y}\right)^{2}\right\}^{-1} .
$$

The boundary conditions (54)-(55) become

$$
\begin{array}{lll}
\zeta \rightarrow-\infty: & \hat{Y}_{1}+\zeta \rightarrow 0, & u-\zeta \rightarrow-\left[\theta_{1}\right], \\
\zeta \rightarrow+\infty: & \hat{Y}_{1} \rightarrow 0, & u \rightarrow 0 .
\end{array}
$$

The problem (60)-(64) can be seen easily to be identical to that describing the structure of the reaction zone of the steady planar flame. Therefore the new eigenvalues $\lambda_{0}$ and $\left[\theta_{1}\right]$ are given in terms of $K$ and $\alpha$ by the relations

$$
\begin{aligned}
& {\left[\theta_{1}\right]=h(K, \alpha),} \\
& \lambda_{0}=\Lambda(K, a) .
\end{aligned}
$$

Then, as a result of (57) and (62), we obtain

$$
\left[Z_{1}\right]=-\frac{h L_{Z}}{(1-\alpha)}
$$

and

$$
\left[\frac{\partial Y_{0}}{\partial x}\right]=\frac{\exp \left\{(1 / 2) \theta_{1}\left(0^{+}, y, t\right)\right\}}{\left\{1+\left(\partial \phi_{0} / \partial y\right)^{2}\right\}^{1 / 2}} .
$$

The jump conditions (58), (65), (67) and (68) are not sufficient for the description of the outer structure; we need an additional jump relation that comes from the analysis, to terms of order $\beta^{-2}$, of (37) within the reaction zone. This gives the equation

$$
\begin{aligned}
& \left(1-\frac{\partial \phi_{0}}{\partial t}\right) \frac{\partial}{\partial \eta}\left(\hat{Y}_{1}+\hat{\theta}_{1}+(1-\alpha) \hat{Z}_{1}\right) \\
& \quad=\left(1+\left(\frac{\partial \phi_{0}}{\partial y}\right)^{2}\right) \frac{\partial^{2}}{\partial \eta^{2}}\left(\hat{Y}_{2}+\hat{\theta}_{2}+(1-\alpha) \frac{\hat{Z}_{2}}{L_{Z}}\right)-l_{e}\left(1+\left(\frac{\partial \phi_{0}}{\partial y}\right)^{2}\right) \frac{\partial^{2} Y_{1}}{\partial \eta^{2}}
\end{aligned}
$$

that can be integrated once to yield

$$
\begin{aligned}
& \left(1-\frac{\partial \phi_{0}}{\partial t}\right)\left(\hat{Y}_{1}+\hat{\theta}_{1}+(1-\alpha) \hat{Z}_{1}\right) \\
& \quad=\left(1+\left(\frac{\partial \phi_{0}}{\partial y}\right)^{2}\right) \frac{\partial}{\partial \eta}\left(\hat{Y}_{2}+\hat{\theta}_{2}+(1-\alpha) \frac{\hat{Z}_{2}}{L_{Z}}-l_{e} \hat{Y}_{1}\right)+N(y, t),
\end{aligned}
$$

where $N(y, t)$ is still unknown. By matching the inner and outer values of the function

$$
\frac{\partial}{\partial x}\left(\frac{Y}{L_{Y}}+\theta+(1-\alpha) \frac{Z}{L_{Z}}\right)
$$


we obtain

$$
\begin{aligned}
& {\left[\frac{\partial}{\partial x}\left(Y_{1}+\theta_{1}+(1-\alpha) \frac{Z_{1}}{L_{Z}}\right)\right]} \\
& \quad=\lim _{\eta \rightarrow \infty} \frac{\partial}{\partial \eta}\left(\hat{Y}_{2}+\hat{\theta}_{2}+(1-\alpha) \frac{\hat{Z}_{2}}{L_{Z}}\right)-\lim _{\eta \rightarrow-\infty} \frac{\partial}{\partial \eta}\left(\hat{Y}_{2}+\hat{\theta}_{2}+(1-\alpha) \frac{\hat{Z}_{2}}{L_{Z}}\right)
\end{aligned}
$$

and using (70) the following jump relation results

$$
\left[\frac{\partial}{\partial x}\left(Y_{1}+\theta_{1}+(1-\alpha) \frac{Z_{1}}{L_{Z}}\right)\right]=l_{e}\left[\frac{\partial Y_{0}}{\partial x}\right]-\left(1-\frac{\partial \phi_{0}}{\partial t}\right)\left(1+\left(\frac{\partial \phi_{0}}{\partial y}\right)^{2}\right)^{-1}\left[\theta_{1}\right]\left(L_{Z}-1\right) .
$$

We can now summarize the results obtained above in the form of the system of equations that describes the response of the flame, for large Zeldovich numbers, in the merged regime.

The concentration of the main reactant and of the intermediate will be described in the outer transport regions by their first approximation: $Y=Y_{0}$ and $Z=Z_{1} / \beta=$ $X h L_{Z} / \beta(1-\alpha)$. They are given by the equations

$$
\begin{aligned}
& \frac{\partial Y}{\partial t}+\left(1-\frac{\partial \phi}{\partial t}\right) \frac{\partial Y}{\partial x}=\Delta Y, \\
& \frac{\partial X}{\partial t}+\left(1-\frac{\partial \phi}{\partial t}\right) \frac{\partial X}{\partial x}=L_{Z}^{-1} \Delta X
\end{aligned}
$$

for $x<0$; while $Y=X=0$ for $x>0$. Here $\phi$ is the first approximation $\phi_{0}$ for the flame displacement. Notice that $X$ is the concentration of the intermediate relative to its value just upstream of the reaction zone, a value that does not change due to unsteady or wrinkling effects, when the activation energies of the two reactions are equal. A similar result was also found by Margolis and Matkowsky in their analysis [9]. When both reactions have different activation energies, the peak value of intermediate changes with unsteady or wrinkling effects [14], because then $K$ changes with flame temperature.

The reduced enthalpy excess $S=Y_{1}+\theta_{1}+(1-\alpha) Z_{1} / L_{Z}$ satisfies, for all $x$, the equation

$$
\frac{\partial S}{\partial t}+\left(1-\frac{\partial \phi}{\partial t}\right) \frac{\partial S}{\partial x}=\Delta\left(S-l_{e} Y-\delta X / L_{Z}\right)
$$

resulting from (45). The parameter $\delta$ is defined by $\delta=h\left(L_{Z}-1\right)$.

These equations must be solved with the boundary conditions

$$
\begin{array}{ll}
x \rightarrow-\infty: & Y-1=X=S=0, \\
x \rightarrow+\infty: & S<\infty, \quad Y=X=0 .
\end{array}
$$

In fact $Y=X=0$ for $x>0$.

In addition the solution must satisfy the following jump conditions at $x=0$ :

$$
\begin{gathered}
{[Y]=[S]=0, \quad[X]=-1,} \\
{\left[\frac{\partial Y}{\partial x}\right]=\left(1+\left(\frac{\partial \phi}{\partial y}\right)^{2}\right)^{-1 / 2} \exp \left(\frac{1}{2} S_{0}\right)} \\
{\left[\frac{\partial S}{\partial x}\right]-l_{e}\left[\frac{\partial Y}{\partial x}\right]=-\delta\left(1-\frac{\partial \phi}{\partial t}\right)\left(1+\left(\frac{\partial \phi}{\partial y}\right)^{2}\right)^{-1}}
\end{gathered}
$$


and where $S_{0}=S(0, y, t)=\theta_{1}\left(0^{+}, y, t\right)$. This model differs from the one associated with one-step Arrhenius kinetics [10] in that it involves the intermediate concentration $X$ in addition to $\phi, Y$ and $S$. The equations for these last variables are coupled with that of $X$, due to the last terms in (75) and $(77 \mathrm{c})$.

The parameter $\delta$ is a measure of the thermal effect of the intermediate species $Z$. If $\delta$ is small the equations for $Y, S$ and $\phi$ become uncoupled from that of $X$. The concentration of the intermediate species can be determined, afterwards, in terms of the flame displacement $\phi$. The stability properties of the flame for $\delta \ll 1$ and its nonlinear response are identical to those of a flame with ordinary Arrhenius kinetics.

If $L_{Z}$ is close to one and $h$ is of order unity, then $\delta \rightarrow 0$. The analysis of Margolis and Matkowsky [8] for the particular case $K=\alpha$, corresponds precisely to this situation because they considered $\left(L_{Z}-1\right)$ of order $1 / \beta$.

If $L_{Z}-1$ is small, but $h$ is large enough so that $\delta$ is of order unity, the previous model simplifies because the equations and boundary conditions for $Y$ and $1-X$, for $x<0$, are identical, so that $X=1-Y$ for $x<0$, with $Y$ given by (73) for $x<0$, and $Y=0$ for $x>0$. The equation for the reduced enthalpy excess $S$ takes in this case the form

$$
\frac{\partial S}{\partial t}+\left(1-\frac{\partial \phi}{\partial t}\right) \frac{\partial S}{\partial x}=\Delta\left(S+\left(\delta-l_{e}\right) Y\right)
$$

to be solved with the boundary conditions of (76), and the jump conditions $[S]=0$ and those given by $(77 \mathrm{~b}, \mathrm{c})$. In this case the simplified model involves only $\phi, S$ and $Y$ as dependent variables.

The value of $h$, or equivalently, the fraction of the intermediate just upstream of the reaction zone, becomes large when $K \ll 1$. In this interesting case the two reactions begin to occur at separated places. As we shall see below, in order to insure stability of the planar flame, $L_{Z}$ in this case must be close to 1 , so that $\delta$ is of order unity.

The system of equations (73)-(77), with appropriate initial conditions, gives, in first approximation for large Zeldovich numbers $\beta$, the nonlinear transient evolution of the flame displacement $\phi$, the concentration of the main reactant $Y$, the intermediate $X$ and the reduced enthalpy excess $S$. The generalization to perturbations involving an additional transverse coordinate is trivial.

The equations for the outer transport zones are written here using the thermaldiffusive model, because the changes in velocity associated with density changes have been neglected. However these hydrodynamic effects could be incorporated in the model by a slight modification of the transport equations, and the addition of the continuity and momentum equations. The jump conditions at the thin flame would not change essentially.

In the resulting limiting model for the unsteady nonplanar flame response, only three parameters enter $L_{Z}, l_{e}=\beta\left(L_{Y}-1\right)$ and the nondimensional concentration $h$ of the intermediate just upstream of the reaction zone. This value $h=h(K, \alpha)$ is calculated in the Appendix by solving numerically the equations for the reaction zone.

5. Linear stability analysis. In the following, we shall give the results of the linear stability analysis of the planar steady flame structure, within the thermal-diffusive model, using directly the model derived above for large values of $\beta$.

The steady planar solution of (73)-(77) can be written as: $\phi_{S}=0$ and

$$
Y_{S}=1-e^{x} \quad X_{S}=e^{x L_{z}}, \quad S_{S}=h\left(e^{x L_{z}}-e^{x}\right)-l_{e} x e^{x}
$$


for $x<0$, and

$$
Y_{S}=X_{S}=S_{S}=0
$$

for $x>0$.

We look for solutions for $Y, X, S$ and $\phi$ in the form

$$
\begin{aligned}
& \phi=a \exp (\omega t+i k y), \\
& Y=Y_{S}+\phi U(x), \\
& X=X_{S}+\phi V(x), \\
& S=S_{S}+\phi W(x),
\end{aligned}
$$

where $a \ll 1$, and the wave number $k$ is real.

When these expressions are taken into the system of (73)-(77), and terms of order $a^{2}$ are neglected, the following dispersion relation is obtained

$$
l_{e}(\Gamma-1-2 \omega)+2 \Gamma^{2}(\Gamma-1)=h \Gamma\left(\Gamma-1+2 \omega\left(L_{Z}-1\right)+2 L_{Z} S_{-}\right)
$$

to insure that the linear problem has nontrivial solutions with $a \neq 0$. Here $\Gamma$ and $S_{\text {- }}$ are given by

$$
\Gamma=\sqrt{1+4\left(\omega+k^{2}\right)}, \quad S_{-}=\frac{1}{2}\left\{L_{Z}-\sqrt{L_{Z}^{2}+4\left(\omega L_{Z}+k^{2}\right)}\right\} .
$$

They must satisfy the requirements

$$
\begin{gathered}
R_{e}(\Gamma) \geqq 1, \\
R_{e}\left(\sqrt{L_{Z}^{2}+4\left(\omega L_{Z}+k^{2}\right)}\right) \geqq L_{Z}
\end{gathered}
$$

although if $L_{Z}>1$ the fulfillment of (82b) implies (82a); if $L_{Z}<1,(82 \mathrm{~b})$ holds if (82a) is satisfied.

The dispersion relation gives $\omega$ in terms of $k$. For the flame to be stable the real part of $\omega$ must be negative for all $k$. In the parameters space $l_{e}, L_{Z}, h$ there is a stable domain, bounded by the stability surface determined by the condition $R_{e}(\omega)=0$, or by the condition of the appearance of a root of $(80)$ with $R_{e}(\omega) \rightarrow \infty$.

We shall begin with a discussion of the stability boundary for the interesting limiting case $h \gg 1$ with $L_{Z}-1 \ll 1$, such that $\delta=h\left(L_{Z}-1\right)$ is of order unity. The dispersion relation takes in this limit the form

$$
l_{e}(\Gamma-1-2 \omega)+2 \Gamma^{2}(\Gamma-1)+\delta(\Gamma-1)(\Gamma-1-2 \omega)=0
$$

involving only the parameters $l_{e}$ and $\delta$. The stability boundary is plotted in Fig. 4 as the closed line $A B C D E A$ bounding the stable domain.

The form of the dispersion relation close to the stability boundary changes along the boundary, and with this the form of the expected response of the flame outside the stable domain. The results are as follows:

a) Along the segment $A C$ the change of stability occurs with $I_{m}(\omega)=0$; in the segment $B C$ there is a nonzero critical wave number $k_{c}$, so that the possibility of cellular flames is suggested. Close to the stability boundary, along the segment $A B$, the dispersion relation, within the thermal diffusive model that we are using, is of the form $\omega=-\left(\left(l_{e}+2\right) / 2\right) k^{2}-(4+\delta) k^{4}$; so that on the unstable side where $l_{e}+2<0$ the possibility of a chaotic response, as found by Sivashinsky [11], is suggested.

b) Along the segment $\mathrm{CE}$, the change of stability occurs with $I_{m}(\omega) \neq 0$. In the segment $E D, k_{c}=0$ and the possibility of pulsating flames is suggested; in the segment $C D, I_{m}(\omega) \neq 0$ and $k_{c} \neq 0$, so the possibility of travelling waves is suggested. 


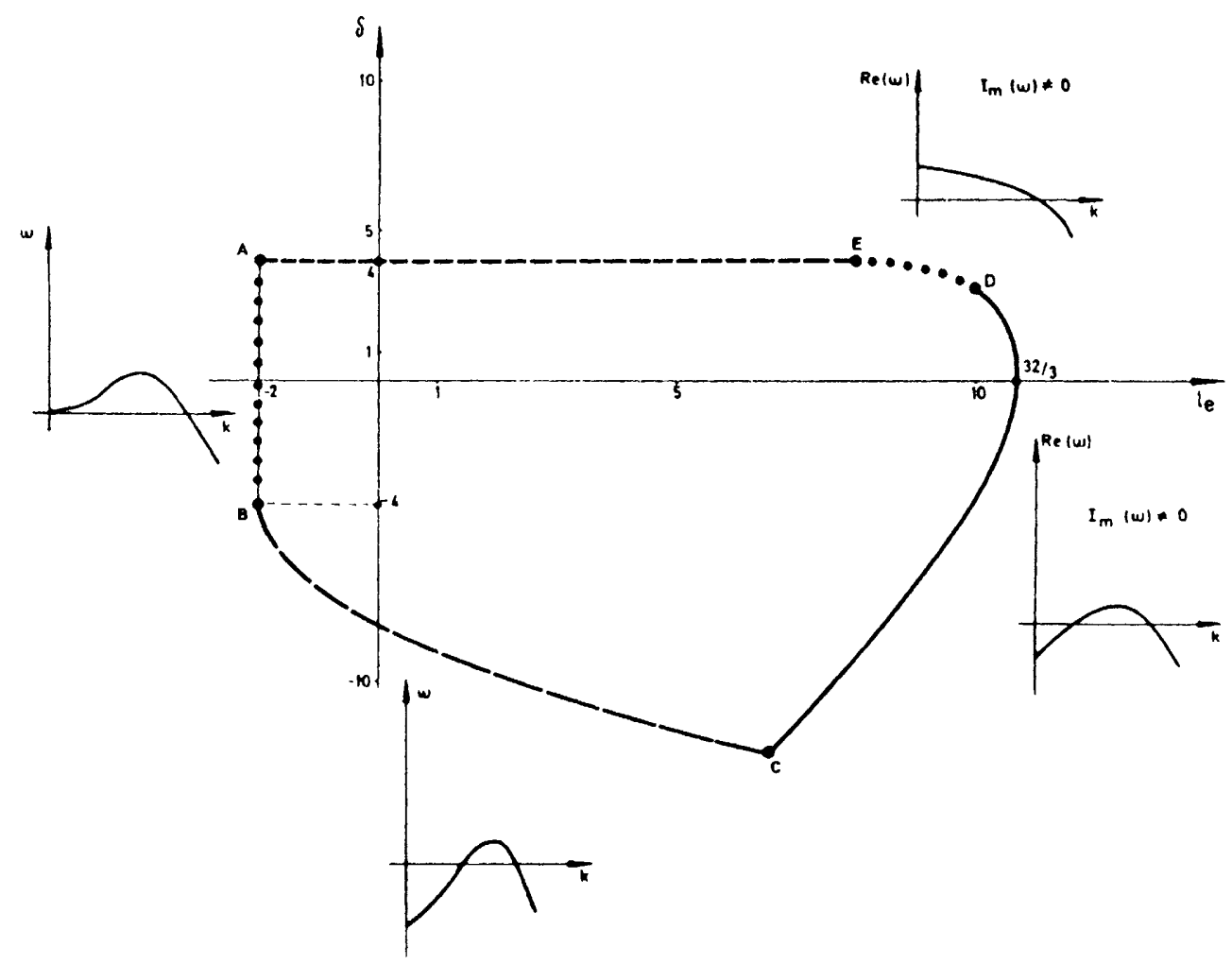

FIG. 4. Stability domain in the $\left(l_{e}, \delta\right)$ plane for $L_{Z}-1 \ll 1$. The form of the dispersion relation, outside the stable domain, is also sketched in the figure.

c) When crossing the line $A E$, corresponding to $\delta=4$, a root of (83) appears with $R_{e}(\omega)>1$, such that $R_{e}(\omega) \rightarrow \infty$ when $\delta-4 \rightarrow 0^{+}$. This instability will only occur for intermediate species with low diffusivity. The appearance of a high frequency response close to this limit implies that, in order to describe the response in an appropriate way, we should retain unsteady effects in the reaction zone. The character of the instability is shown by an analysis, that has been carried out in [14], of the flame stability in the regime of separate reactions zones, when one considers the limiting case when the two reactions zones tend to merge.

These results also hold for the more general case $L_{Z} \neq 1$. We have used the general dispersion relation (80) to calculate the stability boundary in the plane $l_{e}, \delta$ for different values of $L_{Z}$; they are plotted in Fig. 5 . The changes from the previous case are only quantitative.

For example, close to segment $A B^{\prime}$ the dispersion relation takes the form

$$
\omega=-\frac{\varepsilon}{2} k^{2}-\left(4+\frac{\delta\left(L_{Z}+1\right)}{2 L_{Z}^{2}}\right) k^{4},
$$

where $\varepsilon=l_{e}+2$. It is only valid for values of $\delta$ in the interval $4>\delta>-8 L_{Z}^{2} /\left(L_{Z}+1\right)$.

A bifurcation analysis close to the stability boundary $A B^{\prime}$ has been carried out following Sivashinsky [11] to obtain the Kuramoto-Sivashinsky equation

$$
\frac{\partial \psi}{\partial t}-\frac{\varepsilon}{2} \frac{\partial^{2} \psi}{\partial y^{2}}+\left(4+\delta\left(L_{Z}+1\right) / 2 L_{Z}^{2}\right) \frac{\partial^{4} \psi}{\partial y^{4}}+\frac{\varepsilon}{2}\left(\frac{\partial \psi}{\partial y}\right)^{2}=0
$$




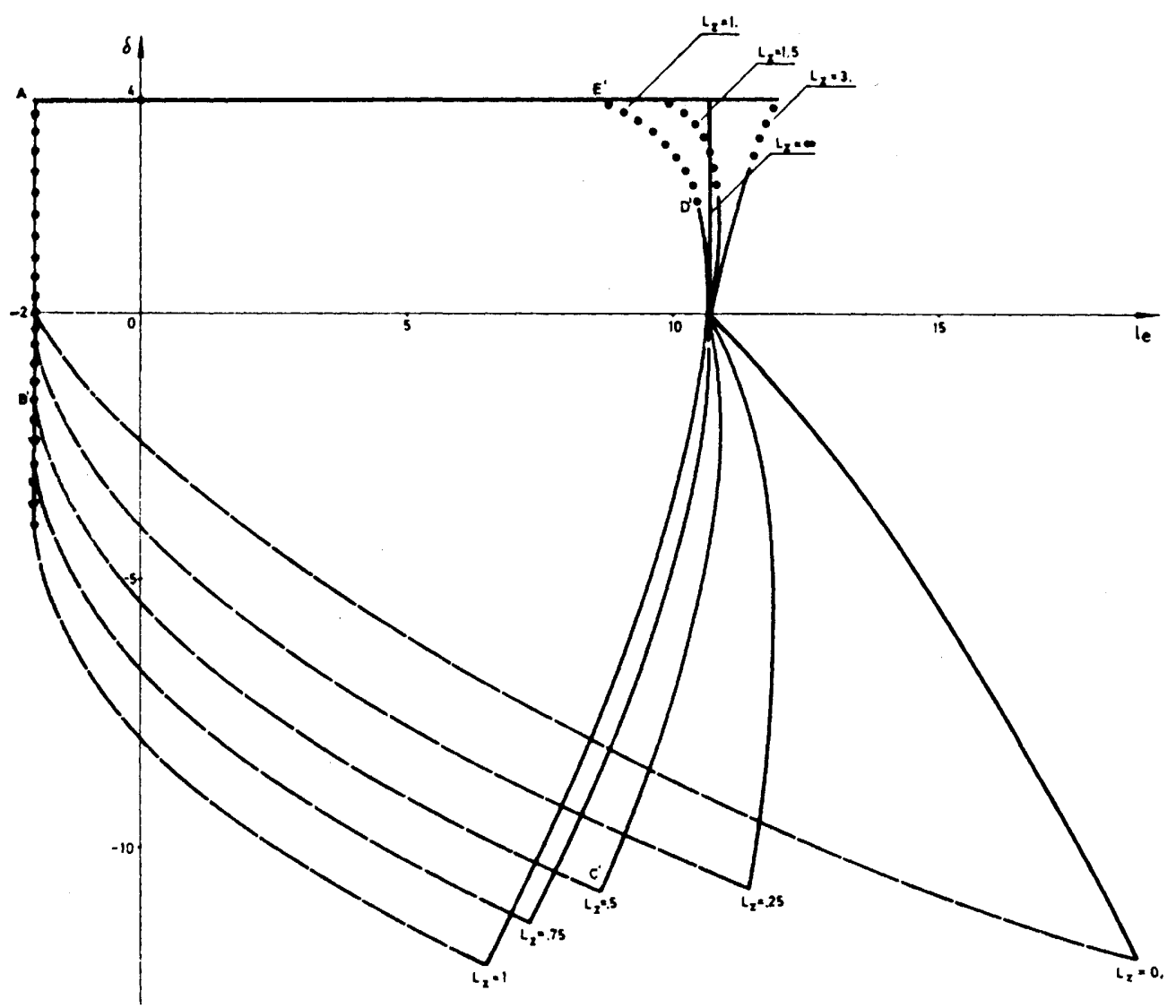

FIG. 5. Stability domain in the $\left(l_{e}, \delta\right)$ plane for different values of $L_{Z}$. Notice that when $L_{Z}>1, \delta>0$ and $\delta<0$ when $L_{Z}<1$.

for the flame displacement $\phi=\varepsilon \psi$. The details of this straight forward analysis are not included here.

As we indicated before, in the limiting case $\delta \rightarrow 0$, associated with $L_{Z} \rightarrow 1$ with $h$ finite, or with $L_{Z}-1$ finite and $h \rightarrow 0$, the stability domain corresponds to the interval $-2<l_{e}<32 / 3$, identical to that of one-step Arrhenius kinetics.

Notice also that the planar flame losses stability at $\delta=h\left(L_{Z}-1\right)=4$ for all positive values of $\left(L_{Z}-1\right)$. The form of the bifurcated solutions close to $\delta=4$ can only be uncovered from a more refined stability analysis retaining unsteady effects in the reaction zone. A stability analysis retaining these effects, in the limiting case $K \ll 1$, has been carried out in [14] and will be published elsewhere.

6. Conclusions. We have carried out an analysis of the structure and stability of flames supported by a simplified mechanism with two sequential reactions, for values of the rate constants such that the reactions are confined to a single thin reaction zone.

One purpose of the analysis is to display the effects of the non-Arrhenius multiple kinetics on the dynamic response of the flame to nonplanar perturbations. When describing the structure of the outer transport zones we have used, for simplicity in the presentation, the thermal-diffusive approximation. Then the response of the flame can be described by a model where only three parameters, $L_{Z}, l_{e}=\left(L_{Y}-1\right) \beta$ and 
$\delta=\left(L_{Z}-1\right) h$, enter. The peak concentration $h$ of the intermediate species that appears here, as well as the steady planar front velocity, proportional to $\Lambda^{-1 / 2}$, have been obtained numerically, in terms of the ratio $K$ of the reaction constants of the two reactions, from the analysis of the thin reaction zone structure; this does not change by unsteady or wrinkling effects. $\Lambda^{-1 / 2}$ and $h$ are shown in Figs. 2 and 3 as functions of $K$ and $\alpha$. Notice that the flame velocity eigenvalue, $\Lambda^{-1 / 2}$, changes from its asymptotic value $\sqrt{2 K} /(1-\alpha)$ when $K \ll 1$, to the value $\sqrt{2}$ for $K \gg 1$. In the limiting cases $K \ll 1$ the two reaction zones separate, and the flame velocity is determined, as found in [5] and [6], by the second reaction; in the limiting case $K \gg 1$ we recover the results associated with an overall Arrhenius kinetics, resulting from the steady state approximation of the intermediate species.

A linear stability analysis of the steady planar flame, carried out with the thermal diffusive approximation shows that, when crossing the stability boundary, we can find chaotic response, cellular flames, travelling waves, and pulsating flames, depending on the values of the three parameters. It is interesting to observe that the stability limit $\left(l_{e}+2\right)=0$ - that appears also for Arrhenius kinetics-and the Markstein length close to this limit are not changed by multiple kinetic effects; however, the size of the stabilizing $k^{4}$ term is dependent on these effects.

If we want to have a stable planar flame, the Lewis number of the main reactant cannot differ much from 1 ; however, the Lewis number $L_{Z}$ of the intermediate species can take values away from unity if the second reaction is fast enough to limit the peak concentration of the intermediate to values of the order of $1 / \beta$.

Even though we have assumed in the analysis that the activation energies of both reactions are equal, the results are valid when the ratio of the activation energies differs from 1 by an amount of order $1 / \beta$; however, it should be noticed that in these cases the value of $K$, and thus the parameter $\delta$ and then the stability properties of the flame, will change when the flame temperature is changed significantly by reactant dilution.

It should be noticed that, within the thermal diffusive model, when crossing the stability limit, the planar flame with ordinary one-step Arrhenius kinetics takes either a chaotic response, if $l_{e}<-2$, or a response involving travelling waves, if $l_{e}>32 / 3$. For the two-step mechanism we may find other instability modes, associated with the diffusive properties of the intermediate species. It is interesting to observe that these instabilities are enhanced for $K \ll 1$, when the two reactions begin to occur in separate zones, and the flame velocity is determined by the second reaction; however, the first reaction determines the peak concentration of the intermediate.

The effects of heat losses on the flame structure and on the stability can be included in the analysis without difficulties, by proceeding as in the analysis of Joulin and Clavin [13]. A heat loss term of order $1 / \beta$ in (13), that will only appear in (75), for the enthalpy excess, in the model, is enough to produce changes by a factor of order 1 in the flame velocity and important changes in the stability properties.

Appendix. For the numerical analysis of the reaction zone structure, it is convenient to work in a phase space, using as independent variable $u=-\tilde{\theta}_{1}$, and

$$
\tilde{Y}_{1}, \quad p=-\frac{1}{\sqrt{\Lambda}} \frac{d \tilde{Y}_{1}}{d \eta}, \quad q=\frac{1}{\sqrt{\Lambda}} \frac{d \tilde{\theta}_{1}}{d \eta}
$$

as dependent variables. The problem of $(28)-(30)-(32)$ then reduces to the solution of the system

$$
q \frac{d p}{d u}=\tilde{Y}_{1} \exp (-u)
$$




$$
\begin{aligned}
& q \frac{d q}{d u}=\left((\alpha-K) \tilde{Y}_{1}+K u\right) \exp (-u), \\
& q \frac{d \tilde{Y}_{1}}{d u}=p
\end{aligned}
$$

with the boundary conditions

$$
\begin{array}{ll}
u=0: & p=q=\tilde{Y}_{1}=0, \\
u \rightarrow \infty: & \tilde{Y}_{1}-u+h \rightarrow 0, \quad p-\frac{1}{\sqrt{\Lambda}} \rightarrow 0, \quad q-\frac{1}{\sqrt{\Lambda}} \rightarrow 0 .
\end{array}
$$

Here $h$ and $\Lambda$ are eigenvalues to be determined as functions of $K$ and $\alpha$.

The point $u=0$, corresponding to the conditions just downstream of the reaction zone, is a singular point. The form of the solution close to this point depends on the value of $K$. For $K>1$, and $u \ll 1$, the solution can be written in the form

$$
\begin{aligned}
& p=\frac{1-K}{\alpha-K} u\left(1+c_{1} u^{r}-u / 3+\cdots\right), \\
& q=u\left(1-c_{1} r u^{r}-\frac{u}{3}+\cdots\right), \\
& \tilde{Y}_{1}=\frac{1-K}{\alpha-K} u\left(1+c_{1} u^{r}+\cdots\right)
\end{aligned}
$$

with $r=K^{1 / 2}-1 ; c_{1}$ is a constant, associated with a nodal character of the point, that must be calculated to insure that for $u \rightarrow \infty$, in the upstream edge of the reaction zone, $p$ and $q$ take the same limiting value $1 / \sqrt{\Lambda}$.

In the case $K<1$ the solution can be written for $u \ll 1$ in the form

$$
\begin{aligned}
& p=c_{2}(1+s) u^{s}+\cdots \\
& q=\frac{u}{s}+c_{2}(\alpha-K) s u^{s}-\frac{u^{2}}{3 s}+\cdots \\
& \tilde{Y}_{1}=c_{2}(1+s) u^{s}+\cdots
\end{aligned}
$$

with $s=1 / \sqrt{K} ; c_{2}$ is a constant that must be calculated to insure that the boundary conditions (A6) are satisfied.

It is not difficult to show that the eigenvalues $\Lambda$ and $h$ satisfy the following symmetry relations, only valid for values of $\alpha$ lower than $K$,

$$
\begin{aligned}
& K \Lambda(K, \alpha)=\Lambda\left(\frac{1}{K}, \frac{\alpha}{K}\right), \\
& h(K, \alpha)=\frac{1-\alpha}{K-\alpha} h\left(\frac{1}{K}, \frac{\alpha}{K}\right)
\end{aligned}
$$

a generalization of a result obtained by Joulin and Clavin [7] for the particular case $\alpha=0$.

The particular case $\alpha=1$ must be analysed independently. In this case from (32) we obtain $\tilde{Y}_{1}=-\tilde{\theta}_{1}$ and then (28) can be integrated once to yield the eigenvalue $\Lambda=\frac{1}{2}$. Afterwards (29) can be integrated to calculate $n(K) ; h \equiv 0$ in this case. For $K \ll 1$, $n \simeq \sqrt{2 / K}+0.655$. 
Asymptotic expressions can be obtained for the eigenvalues $\Lambda(K, \alpha)$ and $h(K, \alpha)$ for large and small values of $K$.

For $K \gg 1$ it is not difficult to show that

$$
\Lambda \simeq \frac{1}{2}+\frac{1-\alpha}{K}, \quad h \approx \frac{1-\alpha}{K} \ln (2 K)
$$

giving errors lower than $10 \%$ if $K \geqq 3$.

The symmetry relations (A7) and (A8) enable us to calculate $\Lambda$ and $h$ in the limit $K \rightarrow 0, \alpha \rightarrow 0$, as

$$
\Lambda \simeq \frac{1}{2 K}+\frac{K-\alpha}{K}, \quad h \simeq(1-\alpha) \ln (2 / K)
$$

to be used only for $\alpha<K$.

In the general limiting case $K \ll 1$ with $1-\alpha$ of order unity, we obtain, using the premixed flame analysis of [12],

$$
\Lambda \simeq \frac{(1-\alpha)^{2}}{2 K}, \quad h \simeq \ln \left(\frac{(1-\alpha)^{2} / K}{\alpha^{2}+1.344 \alpha(1-\alpha)+0.6307(1-\alpha)^{2}}\right) .
$$

One should notice, however, that the asymptotic behaviour (A11) for $K \ll 1$, associated with separated reactions zones, is only valid for very small values of $K$; so that the merged regime covers a very wide range of values of $K$.

The results of the numerical integration, as well as the asymptotic expressions (A9) and (A11) for $\Lambda$ and $h$ as functions of $K$ and $\alpha$, are plotted in Figs. 2 and 3.

\section{REFERENCES}

[1] F. L. Dryer And I. Glassman, High-temperature oxidation of $\mathrm{CO}$ and $\mathrm{CH}_{4}$, Fourteenth Symposium (International) on Combustion, The Combustion Institute, Pittsburgh, 1972, pp. 987-1003.

[2] C. K. WESTBROOK AND F. L. DRYER, Simplified reaction mechanisms for the oxidation of hydrocarbon fuels in flames, Comb. Sci. Techn., 27 (1981), pp. 31-43.

[3] D. A. Frank-Kamenetskil, Diffusion and Heat Transfer in Chemical Kinetics, Chapter VI, Pergamon Press, New York, 1969.

[4] IA. B. Zeldovich, G. I. Barenblatt, V. B. Librovich and G. M. Mahviladze, Mathematical Theory of Combustion and Detonation, Nauka, Moscow, 1980. (In Russian.)

[5] V. S. Berman AND IU. S. Riazantsev, Asymptotic analysis of stationary propagation of the front of a two-stage exothermic reaction in a gas, J. Appl. Math. Mech. (PMM), 37 (1973), pp. 955-1004.

[6] A. K. KAPILA AND G. S. S. LUDFORD, Two-step sequential reactions for large activation energies, Comb. Flame, 29 (1977), pp. 167-176.

[7] G. Joulin AND P. Clavin, Asymptotic analysis of a premixed laminar flame governed by a two-step reaction, Comb. Flame, 25 (1975), pp. 389-392.

[8] S. B. MARGolis AND B. J. MATKOWSKY, Flame propagation with a sequential reaction mechanism, this Journal, 42 (1982), pp. 1175-1188.

[9] —_ Steady and pulsating modes of sequential flame propagation, Comb. Sci. Technol., 27 (1982), p. 193.

[10] B. J. MATKOWSKy AND G. I. Sivashinsky, An asymptotic derivation of two models in flame theory associated with the constant density approximation, this Journal, 37 (1979), pp. 686-699.

[11] G. I. Sivashinsky, Nonlinear analysis of hydrodynamic instability in laminar flames-I. Derivation of basic equations, Acta Astronáutica, 4 (1977), pp. 1177-1206.

[12] A. LiÑAN, The asymptotic structure of counterflow diffusion flames for large activation energies, Acta Astronáutica, 1 (1974), pp. 1007-1039.

[13] G. JOULIN AND P. Clavin, Linear stability analysis of non-adiabatic flames: Diffusional thermal model, Comb. Flame, 35 (1979), pp. 139-153.

[14] J. PelÁEz, Estructura y estabilidad de llamas con cinética química de etapas múltiples, Doctor's thesis, Universidad Politécnica de Madrid, 1984. 